\title{
Combined Inverse-Dynamics/Passivity-Based Control for Robots with Elastic Joints
}

\author{
Andrea Giusti $^{1}$, Jörn Malzahn ${ }^{2}$, Nikolaos G. Tsagarakis ${ }^{2}$, and Matthias Althoff ${ }^{1}$
}

\begin{abstract}
We consider the global tracking control problem of robots with elastic joints. Even if joint elasticity introduces beneficial features for modern applications which require physically resilient and safer robots that can interact with the environment or humans, it challenges the achievable control performance. We propose a novel controller which combines the benefits of two approaches: the intrinsic robustness to model uncertainty from passivity-based control and the implementation efficiency of inverse-dynamics control schemes using a modern recursive algorithm. The novel controller is applied to an elastic-joint reconfigurable robotic arm using a recently proposed framework for on-the-fly control design. Simulation and experimental results validate our proposed approach.
\end{abstract}

\section{INTRODUCTION}

Modern light-weight robots have non-negligible elasticity in the joints that significantly affects the motion-control performance [1], [2]. A typical reason for this elasticity is the use of long shafts, belts and/or harmonic drives in the transmissions [3], [4]. Although formerly the joint elasticity has only been considered as an undesired effect as a source of degradation of the motion-control performance, we have witnessed a growing research interest toward the intentional inclusion of compliant joints in the last two decades. Elasticjoints allow modern robots to overcome limitations of classical rigid robots in advanced applications requiring e.g. safe interaction with the environment, energy storage or force control [5], [6]. However, these new features come at the price of new control challenges.

Control of elastic-joint robots has attracted several researchers. An overview of works up to the mid '90s can be found in [7], [8]. In particular, in [9] the author introduces reasonable assumptions for control design that make the control problem more tractable and allow e.g. the realization of static feedback-linearizing controllers for trajectory tracking. The consideration of the time-scale separation between the (fast) motor-side dynamics and the (slow) link-side dynamics can be found in [10]. That approach has shown to be very effective when sufficiently low elasticity in the joints is present, as described in the experimental comparison with the feedback-linearizing controller on a single-link arm in [11]. A feedforward/feedback method has been introduced in [12] and has also been considered in [2] as an effective,

\footnotetext{
${ }^{1}$ Andrea Giusti and Matthias Althoff are with the Department of Informatics, Technical University of Munich (TUM), Boltzmannstraße, 3, 85748 Garching, Germany. Corresponding email: \{giusti, althoff\}ein.tum.de

${ }^{2}$ Jörn Malzahn and Nikolaos G. Tsagarakis are with the Department of Advanced Robotics, (Fondazione) Istituto Italiano di Tecnologia, via Morego, 30, 16163 Genova, Italy. Corresponding email: \{jorn.malzahn, nikos.tsagarakis\}eit.it
}

practical solution for trajectory tracking, for which however only local stability can be shown. A semi-global tracking controller for elastic-joint robots which interestingly does not require the calculation of the link jerks can be found in [13]. A comparative study of global tracking controllers based on decoupling, backstepping and passivity is described in [14]. Among more recent works, an approach for trajectory tracking where limited sensing capability is considered for lossless elastic-joint manipulators can be found in [15] and a passivity-based approach for both link-side tracking and damping assignment considering nonlinear springs can be found in [16]. For regulation tasks, recent effective approaches can be found e.g. in [17], [18].

We focus on the global tracking control problem. Among the existing methods, the inverse-dynamics (or feedback linearization) control scheme is especially attractive thanks to a recently proposed algorithm that allows its efficient implementation [19]. However, this controller relies on the exact cancellation of the system couplings and may be sensitive to even small model mismatches, thus loosing tracking performance and stability. Such a behaviour is reflected in our simulations and is not new, as other authors have previously realized the risk of poor robustness in that control technique such as in [14], among others. In that paper, the authors propose instead an effective passivitybased controller. On the other hand, an algorithm for a computationally efficient implementation of the passivitybased controllers in [14], [16] as it is for inverse-dynamics control thanks to [19], [20] is not yet available, to the best of our knowledge.

In this work, we provide a controller with better intrinsic robustness with respect to the inverse-dynamics control scheme, which can still be computed in a similar computationally efficient way. Our new global tracking control approach is based on the partial cancellation of the modelled system dynamics combined with the exploitation of the passivity-properties of the robot model. Our proposed combined inverse-dynamics/passivity-based control scheme allows us to merge the enhanced robustness typical of passivity-based controllers (see e.g. [21]-[23]) with the efficient numerical computability recently made possible for inverse-dynamics control of elastic-joint robots in [19], [20]. We test the effectiveness of our controller using simulations and experiments. For the experiments, we consider a modular reconfigurable testbed with elastic joint modules and we implement the on-the-fly synthesis of the model-based global tracking controllers under test according to the framework proposed in [24]. 
The remainder of this paper is structured as follows: we describe in detail the control problem in Sec. II and we recall the essence of the inverse-dynamics control for elastic-joint robots in Sec. III. We introduce our combined inverse-dynamics/passivity-based control law, as well as its efficient implementation in Sec. IV. In Sec. V we evaluate the performance using simulations and experiments.

\section{PROBLEM DESCRIPTION}

Throughout this paper we use bold symbols for matrices and vectors. We omit time dependence of time-varying variables for brevity, except for reasons of clarity. We consider a robot manipulator composed of $N$ links serially connected through elastic joints. By adopting the practically reasonable (so called) "Spong's assumptions" for control design [9], we consider the following dynamical model of a robot arm with $N$ elastic joints:

$$
\begin{array}{r}
\mathbf{M}(\mathbf{q}) \ddot{\mathbf{q}}+\mathbf{n}(\mathbf{q}, \dot{\mathbf{q}})+\mathbf{K}(\mathbf{q}-\theta)=\mathbf{0}, \\
\mathbf{J} \ddot{\theta}+\mathbf{f}(\dot{\theta})+\underbrace{\mathbf{K}(\theta-\mathbf{q})}_{:=\tau_{e}}=\mathbf{u},
\end{array}
$$

where $\mathbf{M}(\mathbf{q}) \in \mathbb{R}^{N \times N}$ is the inertia matrix of the rigid links assembly, $\mathbf{J} \in \mathbb{R}^{N \times N}$ is the constant diagonal matrix of the rotor inertia moments reflected through the square of the respective gear ratio, $\mathbf{K} \in \mathbb{R}^{N \times N}$ is the constant diagonal joint stiffness matrix, $\mathbf{u} \in \mathbb{R}^{N}$ is the vector of input forces/torques, $\mathbf{q} \in \mathbb{R}^{N}$ is the vector of link-side joint position variables, $\theta \in \mathbb{R}^{N}$ is the vector of motor-side joint position variables, $\mathbf{f}(\dot{\theta}) \in \mathbb{R}^{N}$ is the vector of the motor-side friction terms and $\tau_{e} \in \mathbb{R}^{N}$ is the elastic torque vector. In addition, the term $\mathbf{n}(\mathbf{q}, \dot{\mathbf{q}}) \in \mathbb{R}^{N}$ can be expressed as

$$
\mathbf{n}(\mathbf{q}, \dot{\mathbf{q}})=\mathbf{C}(\mathbf{q}, \dot{\mathbf{q}}) \dot{\mathbf{q}}+\mathbf{D} \dot{\mathbf{q}}+\mathbf{g}(\mathbf{q})
$$

where $\mathbf{g}(\mathbf{q}) \in \mathbb{R}^{N}$ is the vector of the gravity terms, $\mathbf{D} \in$ $\mathbb{R}^{N \times N}$ is the matrix of the link-side viscous damping coefficients and $\mathbf{C}(\mathbf{q}, \dot{\mathbf{q}}) \dot{\mathbf{q}} \in \mathbb{R}^{N}$ is the vector of Coriolis and centrifugal terms with $\mathbf{C}(\mathbf{q}, \dot{\mathbf{q}}) \in \mathbb{R}^{N \times N}$ being a matrix such that $\mathbf{N}(\mathbf{q}, \dot{\mathbf{q}})=\dot{\mathbf{M}}(\mathbf{q}, \dot{\mathbf{q}})-2 \mathbf{C}(\mathbf{q}, \dot{\mathbf{q}})$ is skew-symmetric and therefore:

$$
\mathbf{x}^{T} \mathbf{N}(\mathbf{q}, \dot{\mathbf{q}}) \mathbf{x}=\mathbf{0}, \quad \forall \mathbf{x} \in \mathbb{R}^{N}
$$

For the scenario described above we face the problem of designing a control law that guarantees global tracking of sufficiently smooth (at least four times differentiable) linkside joint-space trajectories $\mathbf{q}_{d}(t)$ :

$$
\lim _{t \rightarrow \infty}\left\|\mathbf{q}(t)-\mathbf{q}_{d}(t)\right\|=0 .
$$

To keep the subsequent description succinct, we also omit hereafter joint variable dependencies of the model terms denoting for example the matrix $\mathbf{M}(\mathbf{q})$ by $\mathbf{M}$, its first derivative over time as $\dot{\mathbf{M}}$ instead of $\dot{\mathbf{M}}(\mathbf{q}, \dot{\mathbf{q}})$ and its second derivative as $\ddot{\mathbf{M}}$ instead of $\ddot{\mathbf{M}}(\mathbf{q}, \dot{\mathbf{q}}, \ddot{\mathbf{q}})$. We use the same notation for the other model terms. All norms in this paper are Euclidean norms.

\section{PRELIMINARIES: INVERSE-DYNAMICS CONTROL}

Inverse-Dynamics (ID) control of robot manipulators aim at canceling nonlinear and coupling terms through feedback. With the same goal, the inverse-dynamics control of elasticjoint robots first introduced in [9] is realized by the use of the following control command:

$\mathbf{u}=\mathbf{J K}^{-1}\left[\mathbf{M} \mathbf{y}+2 \dot{\mathbf{M}} \mathbf{q}^{[3]}+\ddot{\mathbf{M}} \ddot{\mathbf{q}}+\ddot{\mathbf{n}}\right]+[\mathbf{M}+\mathbf{J}] \ddot{\mathbf{q}}+\mathbf{n}+\mathbf{f}(\dot{\theta})$,

where $\mathbf{y} \in \mathbb{R}^{N}$ denotes an auxiliary control input and

$$
\ddot{\mathbf{n}}=(\mathbf{C}+\mathbf{D}) \mathbf{q}^{[3]}+\ddot{\mathbf{C}} \dot{\mathbf{q}}+2 \dot{\mathbf{C}} \ddot{\mathbf{q}}+\ddot{\mathbf{g}}
$$

We use the notation of $\mathbf{x}^{[n]}$ for denoting $\frac{d^{n}}{d t^{n}} \mathbf{x}$ with $n \in\{3,4\}$. By rewriting (1) as

$$
\mathbf{M} \ddot{\mathbf{q}}+\mathbf{n}=\mathbf{u}-\mathbf{f}(\dot{\theta})-\mathbf{J} \ddot{\theta}
$$

and by using (3) and the following relation

$$
\ddot{\theta}=\mathbf{K}^{-1}\left[\mathbf{M} \mathbf{q}^{[4]}+2 \dot{\mathbf{M}} \mathbf{q}^{[3]}+\ddot{\mathbf{M}} \ddot{\mathbf{q}}+\ddot{\mathbf{n}}\right]+\ddot{\mathbf{q}}
$$

which is obtained by rearranging and differentiating twice (1a) with respect to time, a linear decoupled system is obtained:

$$
\mathbf{q}^{[4]}=\mathbf{y}
$$

The control law can be completed by assigning an asymptotically stable dynamics of the trajectory tracking error through y such as

$$
\mathbf{y}=\mathbf{q}_{d}^{[4]}-\mathbf{K}_{I D 3} \mathbf{e}^{[3]}-\mathbf{K}_{I D 2} \ddot{\mathbf{e}}-\mathbf{K}_{I D 1} \dot{\mathbf{e}}-\mathbf{K}_{I D 0} \mathbf{e}
$$

where $\mathbf{e}=\mathbf{q}-\mathbf{q}_{d}$ and $\mathbf{K}_{I D i}$ with $i \in\{0,1,2,3\}$ are typically diagonal gain matrices with $K_{I D i}$ being the $j^{\text {th }}$ element of the diagonal, such that

$$
\begin{aligned}
i d_{j}(s):=s^{4}+ & K_{I D 3_{j j}} s^{3}+K_{I D 2_{j j}} s^{2} \\
& +K_{I D 1_{j j}} s+K_{I D 0_{j j}}, \forall j \in\{1, \ldots, N\},
\end{aligned}
$$

are Hurwitz polynomials.

Even though the dependence of model terms from the joint variables and their derivatives in (3) is omitted for brevity, this control law requires measurements (or good estimates) of link-side joint accelerations and jerks. These estimates can be obtained in principle in a model-based fashion using typical sensing capabilities of elastic-joint robots such as $\mathbf{q}$ and $\theta$ (or the elastic torque $\tau_{e}$ instead of either $\mathbf{q}$ or $\theta$ by using the definition of $\tau_{e}$ in (1b)). The first derivative of the position measurements can be obtained by using tachometers or via numerical differentiation as a cheaper practical solution and allows one to obtain $\dot{\tau}_{e}=\mathbf{K}(\dot{\theta}-\dot{\mathbf{q}})$. The joint accelerations and jerks are computed using (1a) and its first derivative:

$$
\begin{gathered}
\ddot{\mathbf{q}}=\mathbf{M}^{-1}\left[\tau_{e}-\mathbf{n}\right], \\
\mathbf{q}^{[3]}=\mathbf{M}^{-1}\left[\dot{\tau}_{e}-(\dot{\mathbf{M}} \ddot{\mathbf{q}}+\dot{\mathbf{n}})\right] .
\end{gathered}
$$




\section{COMBINED INVERSE-DYNAMICS/ PASSIVITY-BASED CONTROL}

As previously introduced, instead of seeking a linear and decoupled system as one does for the inverse-dynamics control method (through perfect cancellation of the overall modelled system dynamics), we aim at only partially canceling nonlinear and coupling terms through feedback and at exploiting the passivity properties of the manipulator dynamics for ensuring stability and control performance. In this section we first present our proposed control law, followed by the description of its efficient implementation.

\section{A. Description of Our Proposed Control Law}

We introduce the combined Inverse-Dynamics/PassivityBased (ID/PB) control law as follows:

$$
\begin{gathered}
\mathbf{u}=\mathbf{J K}^{-1}\left[(2 \dot{\mathbf{M}}+\mathbf{D}) \mathbf{q}^{[3]}+\ddot{\mathbf{M}} \ddot{\mathbf{q}}+\ddot{\mathbf{C}} \dot{\mathbf{q}}+2 \dot{\mathbf{C}} \ddot{\mathbf{q}}+\ddot{\mathbf{g}}\right] \\
-\mathbf{J K} \mathbf{K}^{-1}\left(\mathbf{M} \mathbf{q}_{a}^{[4]}+\mathbf{C} \mathbf{q}_{a}^{[3]}+\Lambda \mathbf{r}\right)+[\mathbf{M}+\mathbf{J}] \ddot{\mathbf{q}}+\mathbf{n}+\mathbf{f}(\dot{\theta}),
\end{gathered}
$$

where

$$
\begin{gathered}
\mathbf{r}=\mathbf{e}^{[3]}+\mathbf{K}_{H Y 3} \ddot{\mathbf{e}}+\mathbf{K}_{H Y 2} \dot{\mathbf{e}}+\mathbf{K}_{H Y 1} \mathbf{e}, \\
\mathbf{q}_{a}^{[3]}=-\mathbf{q}_{d}^{[3]}+\mathbf{K}_{H Y 3} \ddot{\mathbf{e}}+\mathbf{K}_{H Y 2} \dot{\mathbf{e}}+\mathbf{K}_{H Y 1} \mathbf{e}, \\
\mathbf{q}_{a}^{[4]}=-\mathbf{q}_{d}^{[4]}+\mathbf{K}_{H Y 3} \mathbf{e}^{[3]}+\mathbf{K}_{H Y 2} \ddot{\mathbf{e}}+\mathbf{K}_{H Y 1} \dot{\mathbf{e}} .
\end{gathered}
$$

Applying the control law of (10) to the system in (5) with (6) and (4) yields after algebraic manipulation to

$$
\mathbf{M} \dot{\mathbf{r}}+\mathbf{C r}+\Lambda \mathbf{r}=\mathbf{0} .
$$

In light of (11), the trajectory tracking error will globally converge to zero for a positive definite matrix $\Lambda$, provided that the diagonal gain matrices $\mathbf{K}_{H Y i} \forall i \in\{1,2,3\}$, with $K_{H Y i_{j j}}$ being the $j^{\text {th }}$ elements of the diagonals, are selected so that

$$
\begin{aligned}
h y_{j}(s):=s^{3}+K_{H Y 3_{j j}} s^{2}+ & K_{H Y 2_{j j} s} s \\
& +K_{H Y 1_{j j}}, \forall j \in\{1, \ldots N\},
\end{aligned}
$$

are Hurwitz polynomials. This can be seen by considering the general result for passivity-based control in [25, Theorem 4.1]. In particular, it is worth mentioning that when disturbances and model mismatches are considered, a perturbation term $\psi$ arises and the closed loop dynamics in (11) can be similarly written as

$$
\mathbf{M} \dot{\mathbf{r}}+\mathbf{C r}+\Lambda \mathbf{r}=\psi .
$$

Now, it can be noticed that (13) represents the classical structure that is typically desired when developing passivitybased tracking controllers. Indeed, by considering the storage function $V=\frac{1}{2} \mathbf{r}^{T} \mathbf{M r}$, it defines an output strictly passive operator $\psi \rightarrow \mathbf{r}$ [26, Lemma 2.7]. It is additionally worth mentioning that the proposed controller is still applicable when the manipulator is composed of mixed rigid/elastic joints with the use of dynamic feedback terms, as described in [27] where the applicability of the inverse-dynamics control scheme is considered.

\section{B. Efficient Implementation of the Control Law}

Our proposed controller can be efficiently implemented by combining the use of a recently introduced recursive Newton-Euler algorithm for elastic joint robots (EJNEA) in [19] with the use of a modified recursive Newton-Euler algorithm ( $\mathrm{NE}^{*}$ ) for passivity-based control of rigid robot manipulators in [21]. To describe how to properly combine these algorithms, let us denote by $\operatorname{EJNEA}\left(\mathbf{q}, \dot{\mathbf{q}}, \ddot{\mathbf{q}}, \mathbf{q}^{[3]}, \mathbf{q}^{[4]}\right)$ the algorithm that provides the following relation as proposed in [19]:

$$
\begin{aligned}
\mathbf{J K}^{-1}\left[\mathbf{M q}^{[4]}+2 \dot{\mathbf{M}} \mathbf{q}^{[3]}+\ddot{\mathbf{M}} \ddot{\mathbf{q}}+\ddot{\mathbf{n}}\right]+[\mathbf{M}+\mathbf{J}] \ddot{\mathbf{q}}+\mathbf{n} \\
=\operatorname{EJNEA}\left(\mathbf{q}, \dot{\mathbf{q}}, \ddot{\mathbf{q}}, \mathbf{q}^{[3]}, \mathbf{q}^{[4]}\right),
\end{aligned}
$$

and by $\mathrm{NE}_{0}^{*}\left(\mathbf{q}, \dot{\mathbf{q}}, \dot{\mathbf{q}}_{a}, \ddot{\mathbf{q}}\right)$ the algorithm that provides the following relation as proposed in [21]:

$$
\mathbf{M} \ddot{\mathbf{q}}+\mathbf{C} \dot{\mathbf{q}}_{a}=\mathrm{NE}_{0}^{*}\left(\mathbf{q}, \dot{\mathbf{q}}, \dot{\mathbf{q}}_{a}, \ddot{\mathbf{q}}\right) .
$$

We would like to remind the unfamiliar reader that model terms such as $\ddot{\mathbf{M}}$ or $\ddot{\mathbf{n}}$ among others may contain hundreds of terms and therefore the above mentioned algorithms are crucial for efficient numerical computations, especially when the number of joints $N$ becomes large. With these two algorithms at hand, our proposed control law can be efficiently implemented as follows

$$
\begin{aligned}
\mathbf{u}= & \operatorname{EJNEA}\left(\mathbf{q}, \dot{\mathbf{q}}, \ddot{\mathbf{q}}, \mathbf{q}^{[3]}, \mathbf{0}\right) \\
& -\mathbf{J K}^{-1}\left(\operatorname{NE}_{0}^{*}(\mathbf{q}, \dot{\mathbf{q}}, \underbrace{\mathbf{q}_{a}^{[3]}+\mathbf{q}^{[3]}}_{=\mathbf{r}}, \mathbf{q}_{a}^{[4]})+\Lambda \mathbf{r}\right)+\mathbf{f}(\dot{\theta}) .
\end{aligned}
$$

Once $\ddot{\mathbf{q}}$ and $\mathbf{q}^{[3]}$ are computed (see e.g. [19, Sec. IV]) which inverse-dynamics control also requires, the complexity for the computation of (14) is linear in the number of joints $(\mathscr{O}(N))$, since both $\operatorname{EJNEA}\left(\mathbf{q}, \dot{\mathbf{q}}, \ddot{\mathbf{q}}, \mathbf{q}^{[3]}, \mathbf{q}^{[4]}\right)$ and $\mathrm{NE}_{0}^{*}\left(\mathbf{q}, \dot{\mathbf{q}}, \dot{\mathbf{q}}_{a}, \ddot{\mathbf{q}}\right)$ have linear computational complexity.

Remark 1: The use of passivity-based control principles allows us to introduce practical benefits in terms of intrinsic robustness as is typical for passivity-based controllers, thus avoiding the complete cancellation of the overall modelled system dynamics. A more detailed discussion of the benefits introduced by the consideration of passivity properties for controlling mechanical systems can be found e.g. in [22], [26].

Remark 2: Our proposed control law is a passivity-based global tracking controller for elastic-joint robots with efficient numerical computability by means of recursive NE algorithms. Previously proposed passivity-based global tracking controllers (see e.g. [14], [16]) would require instead the availability of the matrices corresponding to the first and second derivatives of $\mathbf{C}$ and $\mathbf{M}$ for which the property in (2) holds. In our approach these terms are not necessarily required thanks to the use of the above mentioned recursive algorithms. In fact, according the proposed efficient implementation of the control law, the contributions of $\dot{\mathbf{M}}, \ddot{\mathbf{M}}, \dot{\mathbf{C}}$, $\ddot{\mathbf{C}}$, are already included in the numerical computations of EJNEA. 
Remark 3: The tuning phase of our proposed control law is more practical and intuitive (as experienced in our simulations and experiments) thanks to the introduction of the gain matrix $\Lambda$ and the order reduction by one of the polynomials that has to be maintained Hurwitz (compare (12) with (7)). In practice the $\Lambda$ gain matrix has an intuitive influence on the tracking error since it allows one to directly inject damping in (13). This results in an intuitive tuning knob that allows us to reduce the tracking error when increasing the matrix entries, without having to consider the roots of a polynomial for a required increase of tracking performance. This will become more evident in Fig. 3 of the next section.

\section{EVALUATION OF THE TRACKING PERFORMANCE}

In this section we evaluate the tracking performance of our proposed control law with respect to the inverse-dynamics controller and its sensitivity to small mismatches of the system dynamics. We first describe a practical solution that we adopted for our experimental setup to obtain filtered acceleration and jerk estimates. Successively, we describe the simulation testbed and simulation results, to conclude this section with the description of the experimental testbed and experimental results.

\section{A. Estimation of Joint Accelerations and Jerks}

The noise of the available torque sensor results in noisy estimations of the joint accelerations and jerks by means of (8) and (9). To reduce the noise of these estimates, we implement a practical solution following the idea of the kinematic Kalman filter proposed in [28]. We consider availability of measurements (or estimations) of joint positions, velocities and accelerations. While in general joint position measurements are obtained using encoders and the velocity measurements can be obtained using tachometers (or numerical differentiation of position measurements), an estimation of the joint accelerations can be obtained in a model-based way as previously recalled using (8). Given $\mathbf{x}(t)=\left[\begin{array}{llll}q & \dot{q} & \ddot{q} & q^{[3]}\end{array}\right]^{T}$, we consider the following model for designing the filter:

$$
\dot{\mathbf{x}}(t)=\mathbf{A} \mathbf{x}(t)+\Gamma w(t), \quad \mathbf{y}(t)=\mathbf{C} \mathbf{x}(t)+\mathbf{e}(t),
$$

where $w(t)$ is the unbiased white process noise with covariance $c, \mathbf{y}(t)$ is the vector of the available measurements and $\mathbf{e}(t)$ is the vector of unbiased white measurement noise having covariance matrix $\mathbf{R}$. The system matrices that we consider are

$$
\mathbf{A}=\left[\begin{array}{llll}
0 & 1 & 0 & 0 \\
0 & 0 & 1 & 0 \\
0 & 0 & 0 & 1 \\
0 & 0 & 0 & 0
\end{array}\right], \Gamma=\left[\begin{array}{l}
0 \\
0 \\
0 \\
1
\end{array}\right], \mathbf{C}=\left[\begin{array}{llll}
1 & 0 & 0 & 0 \\
0 & 1 & 0 & 0 \\
0 & 0 & 1 & 0
\end{array}\right]
$$

Similarly to the considerations made in [29] for $\ddot{q}$, within this model $w(t)$ represents a surrogate of $q^{[4]}$ and its covariance $c$ can be practically regarded as a filter-tuning parameter since the above mentioned stochastic model may not approximate accurately the actual robot motion. For our testbed, while the position measurements and the velocity estimates are provided by the motor driver boards, the noisy acceleration estimates are obtained using (8). After obtaining the covariance matrix $\mathbf{R}$ for the available measurements/estimations and selecting the parameter $c$, we implement the discrete time version of this kinematic Kalman filter using the Matlab's functions $c 2 d$, dleq and destim.

\section{B. Simulation Results}

We consider a simple $2 \mathrm{R}$ and a $6 \mathrm{R}$ planar robot composed of cylindrical links and working in the vertical plane. For $i \in\{1,2, \ldots 6\}$, the nominal relevant parameters are the lengths of the links $l_{i}=0.5 \mathrm{~m}$, the radius of the cylindrical links $r_{i}=0.025 \mathrm{~m}$ and their mass $m_{i}=2.5 \mathrm{~kg}$. The relevant parameters for the model of the motor-side dynamics are the rotor inertias $J_{i}=0.5 \mathrm{kgm}^{2}$ and the elastic-joint stiffnesses $K_{i}=4650 \mathrm{Nm} / \mathrm{rad}$. We consider a sampling time of $1 \mathrm{~ms}$.

We show the sensitivity to model mismatches by simulating small mismatches of the knowledge of the mass (and inertia) at the last link of the respective arms. The desired trajectory has been designed using subsequent smooth $\sin ^{2}$ profiles as in [30, Section 6.3], setting maximum acceleration at $3 \mathrm{rad} / \mathrm{s}^{2}$ and maximum velocity at $1.5 \mathrm{rad} / \mathrm{s}$. The resulting trajectory for each joint is shown in Fig. 1.

The simulation results for the $2 \mathrm{R}$ robot are collected in Fig. 2. As shown in this figure, for the case of $5 \%$ and $10 \%$ of unexpected additional mass at the second link, our proposed controller has a remarkable insensitivity with respect to the ID control method, leading to less tracking error and a smoother behaviour. The gains of the two controllers have been properly tuned for a fair comparison by allowing the tracking error to be comparable in the case of perfect model information as it is noticeable from the first column of plots in Fig. 2. It is worth stressing that sampling effects have been considered in these simulations (sampling time $1 \mathrm{~ms}$ ). This introduces a small perturbation that prevents the tracking error to approach the numerical precision of the solver as one would otherwise expect for the case of perfect knowledge of the model without sampling effects included. The gains that have been used for ID control and for combined ID/PB control are respectively: $\mathbf{K}_{I D 3}=150 \mathbf{I}$, $\mathbf{K}_{I D 2}=7 \cdot 10^{3} \mathbf{I}, \mathbf{K}_{I D 1}=140 \cdot 10^{3} \mathbf{I}, \mathbf{K}_{I D 0}=1 \cdot 10^{6} \mathbf{I}$ and $\mathbf{K}_{H Y 3}=150 \mathbf{I}, \mathbf{K}_{H Y 2}=6,5 \cdot 10^{3} \mathbf{I}, \mathbf{K}_{H Y 1}=40 \cdot 10^{3} \mathbf{I}, \Lambda=9 \mathbf{I}$, where $\mathbf{I}$ is the identity matrix of proper dimension.

We show the effect of the increase of the elements of $\Lambda$ on the tracking performance for the combined ID/PB controller in Fig. 3. This figure shows the intuitive effect

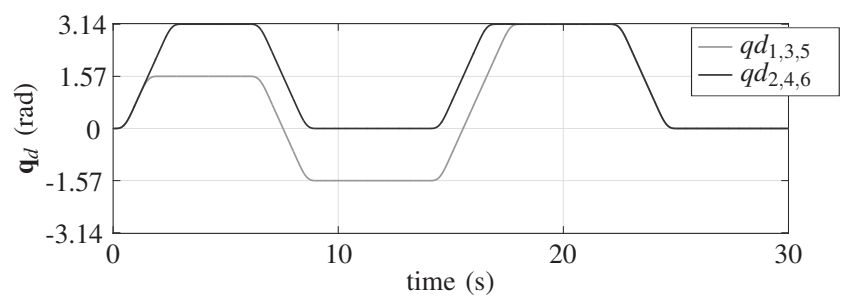

Fig. 1. Required joint-space trajectory for the simulations and experiments shown in this paper. 


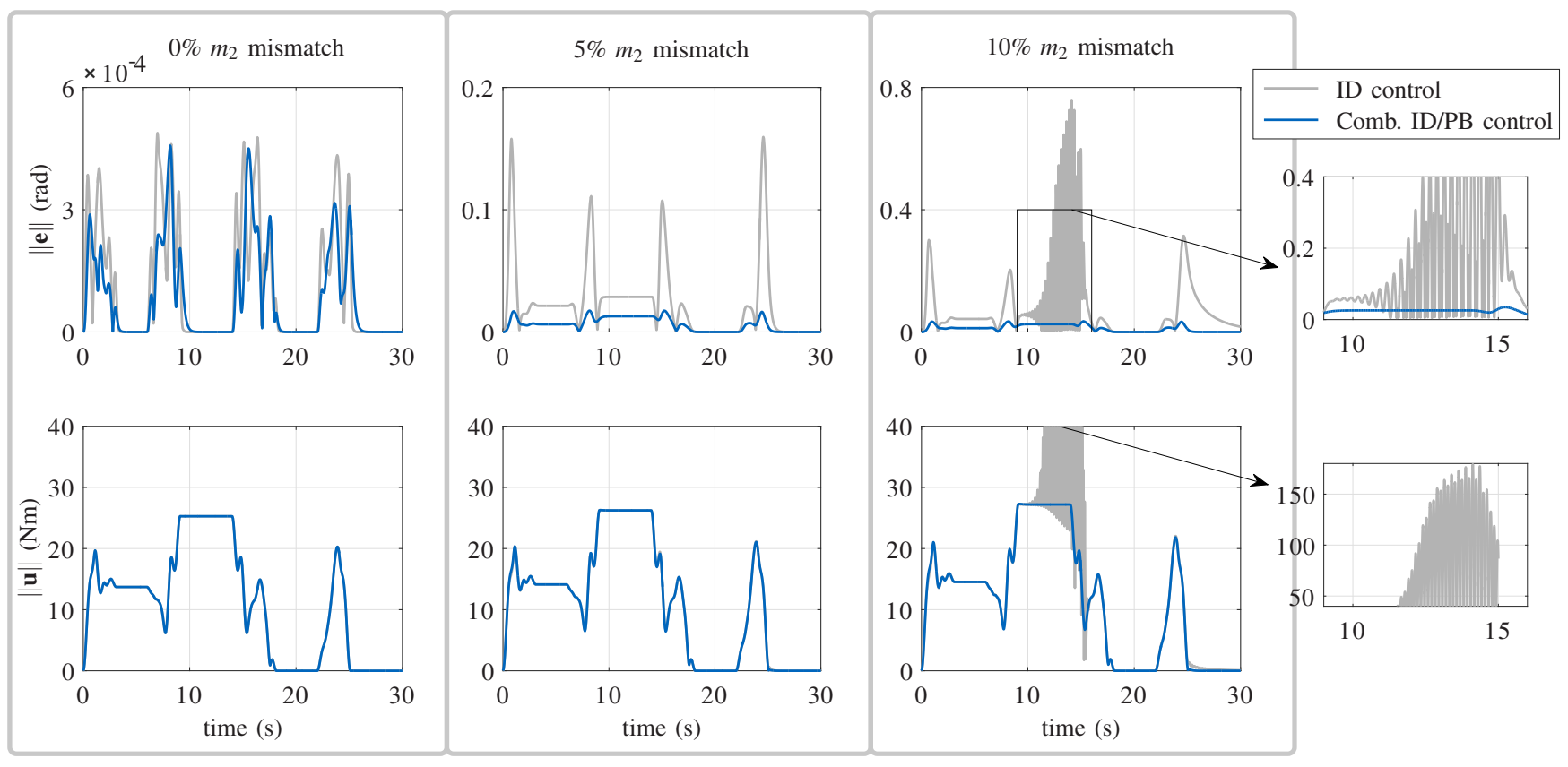

Fig. 2. Simulation comparison of tracking performance and torque commands between ID control and combined ID/PB control, for a $2 \mathrm{R}$ planar arm. The simulations have been performed considering perfect model information (first column of plots), an additive 5\% mismatch for the second link mass (second column of plots), and finally an additive 10\% mismatch for the second link mass (third column of plots).

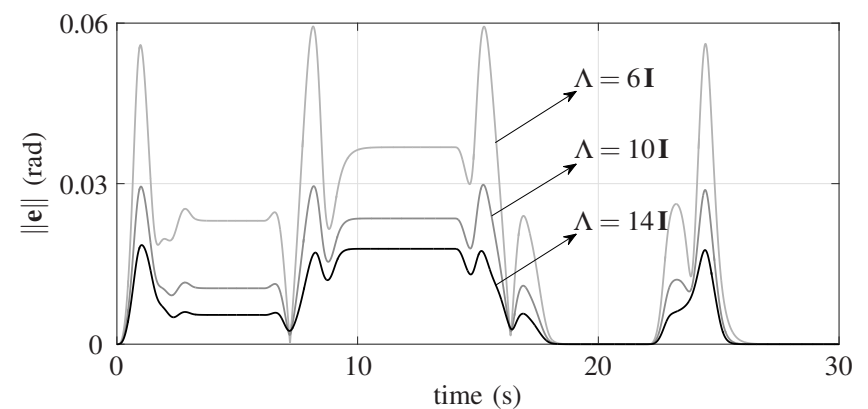

Fig. 3. Effect of $\Lambda$ on the tracking performance of the combined ID/PB controller for a $2 \mathrm{R}$ planar arm, considering an additive $10 \%$ mismatch for the second link mass and keeping fixed the other gain matrices.

of reduction of the tracking error when simply increasing the matrix entries (keeping fixed the other gain matrices). The same gain matrices $\mathbf{K}_{H Y 1}, \mathbf{K}_{H Y 2}$ and $\mathbf{K}_{H Y 3}$ mentioned previously have been used for these simulations.

To investigate the effect of the observer for estimating joint accelerations and jerks that our available experimental testbed requires, we include in the simulations the observer and position/velocity measurement noise such that the following covariance matrix is used $\mathbf{R}=\operatorname{diag}\left\{10^{-9}, 10^{-7}, 20\right\}$. We show the results of these simulations in the second and third row of plots of Fig. 4, where the tracking performance of a $2 \mathrm{R}$ planar robot (first column of plots) and of a $6 \mathrm{R}$ planar robot (second column of plots) are presented, all with $10 \%$ mass mismatch at the last link. From this figure, we can infer that the introduction of the observer significantly robustifies the ID controller in the presence of model mismatches so that the performance for the $2 \mathrm{R}$ robot become comparable. In the second and third row of plots in Fig. 4 we also show the effect of the reduction of the tuning parameter $c$ of the filter. The reduction of this parameter is required in practice when one wishes to increase noise suppression from the measurements at the price of an unavoidable phase lag. With the increase of the complexity of the simulated robot from $2 \mathrm{R}$ to $6 \mathrm{R}$ (see second column of plots in Fig. 4) the simulations show that the ID controller is more sensitive to the phase-lag introduced by the observer with respect to the combined ID/PB controller, which is therefore still preferable. The gains that have been used for ID control and for combined ID/PB control for these simulations are the same as the previously mentioned ones.

\section{Experimental Results}

Our experimental robot testbed is composed of aluminum profiles (used as link-modules) and elastic-joint-modules ensembles. Thanks to the modularity of the available setup we are able to quickly modify the robot assembly. Among the possible assemblies that can be obtained with our reconfigurable testbed, two of them are shown in Fig. 5 and Fig. 6 where different link-modules have been used. The modular and reconfigurable nature of this set-up allows us to apply a framework for automatically designing model-based controllers of modular robot manipulators [24]. Following that approach, each module is systematically characterized and a compact set of information (module data) is stored within the module or in a database. These data contain a unique identification number as well as parameters which characterize the kinematics and dynamics of the module itself. Once the manipulator is assembled using a set of possibly heterogeneous modules, the module data are col- 


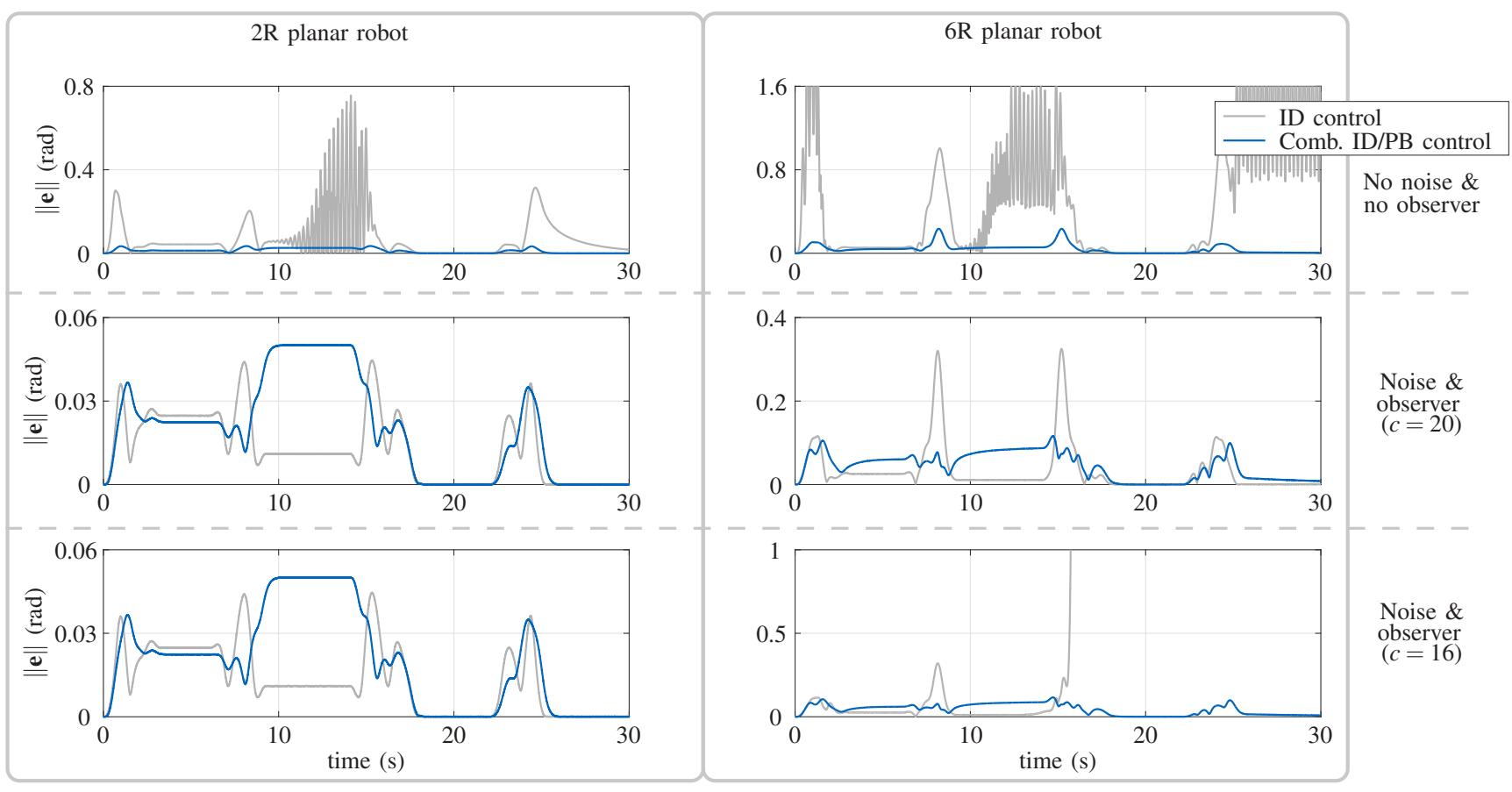

Fig. 4. Simulation comparison showing the effect of the observer for a $2 \mathrm{R}$ planar robot (left column of plots) and for a $6 \mathrm{R}$ planar robot (right column of plots), both working in the vertical plane. All plots represent a simulation with $10 \%$ added mass mismatch at the last link of each arm. The first row of plots collects results for a scenario without noise and without observer in use, the second row considers measurement noise and the use of the observer with $c=20$ and the last row considers measurement noise and the use of the observer with $c=16$.

lected at a central control unit, which processes them for automatically obtaining the kinematic and dynamic parameters of the resulting manipulator and model-based controllers. The consideration of joint modules that introduce elasticity requires that in addition to the data for obtaining the rigid links assembly dynamics [24] and the rotor inertia (with gear ratio), also the elastic-joint stiffness is stored. Once the description of the robot is obtained by processing the module data as described in [24], the efficient implementation of our proposed combined ID/PB control law can be done as previously described in Sec. IV-B.

The available measurements provided by the motor driver boards are the motor-side joint positions, the deflection of the elastic elements $(\Delta)$ and the rate of change of these quantities $(\dot{\theta}, \dot{\Delta})$. The link-side joint positions and velocities are obtained using $\mathbf{q}=\theta+\Delta$ and $\dot{\mathbf{q}}=\dot{\theta}+\dot{\Delta}$. The knowledge of the joint stiffness allows us to obtain the estimate of the elastic torques $\tau_{e}=\mathbf{K}(\theta-\mathbf{q})$ and its rate of change $\dot{\tau}_{e}=$ $\mathbf{K}(\dot{\theta}-\dot{\mathbf{q}})$. For implementing our proposed control approach, we use Simulink Real Time 2015b and an embedded target PC (PC-104) equipped with 2 GB RAM and an Intel Core 2 Duo CPU running at $1.86 \mathrm{GHz}$. The sampling time is $1 \mathrm{~ms}$. We obtain the kinematic and dynamic parameters of the modules using CAD data. The motor-side friction parameters of the joint modules as well as the rotor inertia have been estimated by implementing a simple identification procedure for each motor alone, using a least square approach and the following model that neglects link-side inertia: $J_{i} \ddot{\theta}_{i}+$ $\beta_{v} \dot{\theta}+\beta_{c} \operatorname{sign}(\dot{\theta})=u_{i}$, for the $i^{t h}$ joint, where $\beta_{v}$ and $\beta_{c}$ are the viscous and static friction coefficient. The elastic-joint stiffness and the rotor inertia through the square of the gear ratio that we have estimated are not significantly different from those we described for our simulation testbed.

After assembly, the controller is automatically generated by processing the module data and by efficiently implementing our proposed control law as described in Sec. IVB. We validate the automatically obtained robot models by measuring the elastic joint torques $\tau_{e}$ while the robot performs movements and we finally compare them with the torques computed from the model. Fig. 5 and Fig. 6 show the quality of the match between the model and the actual torque measurements, where two different robot assemblies are respectively considered among the several we tested (which all have comparable results). These figures show a significantly good match during the motion.

The experimental results of the tracking performance of both the ID and the combined ID/PB control using the assembly of Fig. 5 are presented in Fig. 7. We show the effect of a model mismatch by testing both the plain robot (first column of plots of Fig. 7) and the arm with an additional $2 \mathrm{~kg}$ load at the end of the second link, which is unknown for control design (second column of plots of Fig. 7). The tuning has been performed such that both controllers provide comparable results in terms of tracking performance and amplification of the measurement noise for the plain robot (see first column of Fig. 7) although significant tuning effort was required to make the ID controller perform as good as the combined ID/PB.

It is worth mentioning that the tracking performance is acceptable even when considering the loaded robot for both 

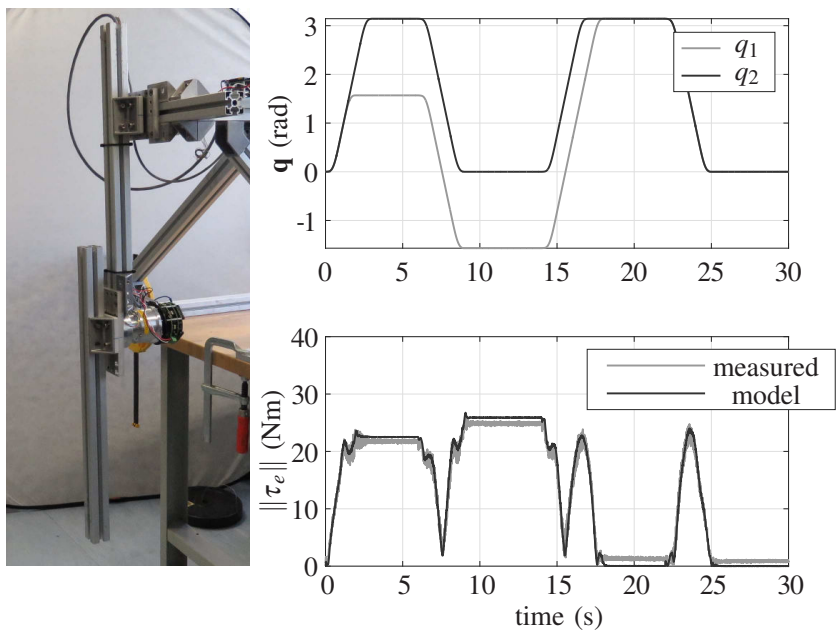

Fig. 5. Validation of the automatically generated model for assembly I with total moving mass of about $7 \mathrm{~kg}$.

controllers (second column of plots in Fig. 7), which is consistent with our simulation results for the $2 \mathrm{R}$ robot case when using the observer. With our experimental testbed the use of the observer is necessary for noise suppression. The main purpose of our experiments, however, is to show realworld applicability of our proposed controller. It should be noted that the use of the described observer does not necessarily maintain the global tracking properties of the control law. Therefore, the accelerations and jerks estimation only using (8) and (9) should be preferred, as long as torque sensing is sufficiently precise. In such a scenario, global tracking is ensured as supported by theory and we believe that the clear benefits shown in simulations e.g. in Fig. 2 would reasonably appear also in practice. It is also important to report that no significant computational overhead has been experienced by our proposed controller compared to the ID control as shown in Tab. I.

\section{CONCLUSION}

A new global tracking control law for elastic-joint robots with enhanced insensitivity to model uncertainty is proposed. The use of passivity-based control concepts allows us to avoid the complete inversion of the system dynamics through feedback. The proposed controller is rather easy to tune and is a passivity-based global tracking controller for robots with elastic joints which can be efficiently implemented by means of recursive NE algorithms. Simulation and experimental results show its validity and its computational efficiency. In addition, as a result of applying the approach of [24] to the available setup, we implement for the first time the automatic generation of model-based tracking controllers for an elasticjoint reconfigurable robot.

Our proposed controller can be implemented using standard sensing capabilities of robot manipulators (encoders and possibly tachometers). The precision of these measurements is crucial for avoiding highly noisy estimates of the joint accelerations and jerks through the use of the elastic-joint torques and respective derivatives. However, an effective
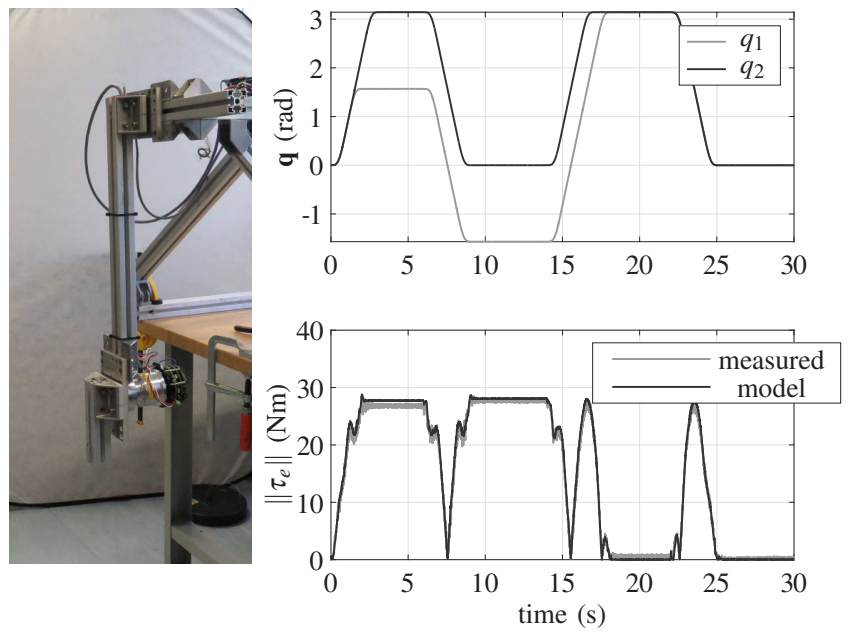

Fig. 6. Validation of the automatically generated model for assembly II with total moving mass of about $6.1 \mathrm{~kg}$.

TABLE I

TOTAL EXECUTION TIME OF THE CONTROLLERS IN THE EXPERIMENTS.

\begin{tabular}{lcc}
\hline & ID control & Comb. ID/PB control \\
\hline Max. $\left(10^{-4} \mathrm{~s}\right)$ & 1.445 & 1.449 \\
Mean $\left(10^{-5} \mathrm{~s}\right)$ & 5.32 & 5.39 \\
Std. dev. $\left(10^{-5} \mathrm{~s}\right)$ & 2.61 & 2.63 \\
\hline
\end{tabular}

filtering solution has been proposed in the event that high precision torque sensing is not available and related interesting robustifying effects have been discussed.

We have not yet realized an experimental testbed with a more complex structure since different hardware has not yet been available. An extension of this work with the application of the combined ID/PB controller to a more complex robotic arm with series elastic actuators will be subject of upcoming work. We also plan to perform a more detailed sensitivity analysis with respect to external disturbances and parametric model uncertainty.

\section{ACKNOWLEDGMENT}

The research leading to these results has received funding from the People Programme (Marie Curie Actions) of the European Union's Seventh Framework Programme FP7/20072013/ under REA grant agreement number 608022.

\section{REFERENCES}

[1] M. C. Good, L. M. Sweet, and K. L. Strobel, "Dynamic models for control system design of integrated robot and drive systems," Journal of Dynamic Systems, Measurement, and Control, vol. 107, no. 1, pp. 53-59, Mar. 1985.

[2] A. Albu-Schäffer, C. Ott, and G. Hirzinger, "A unified passivitybased control framework for position, torque and impedance control of flexible joint robots," Int. J. Robot. Res., vol. 26, no. 1, pp. 23-39, 2007.

[3] T. D. Tuttle, "Understanding and modeling the behaviour of a harmonic drive gear transmission," MIT Artificial Intelligence Laboratory, Tech. Rep. 1365, May 1992.

[4] A. De Luca and W. Book, Handbook of Robotics. Springer, 2008, book section Robots with Flexible Elements, pp. 287-320.

[5] G. A. Pratt and M. M. Williamson, "Series elastic actuators," in Proc. IEEE/RSJ Int. Conf. on Intelligent Robots and Systems, vol. 1, Aug. 1995, pp. 399-406. 


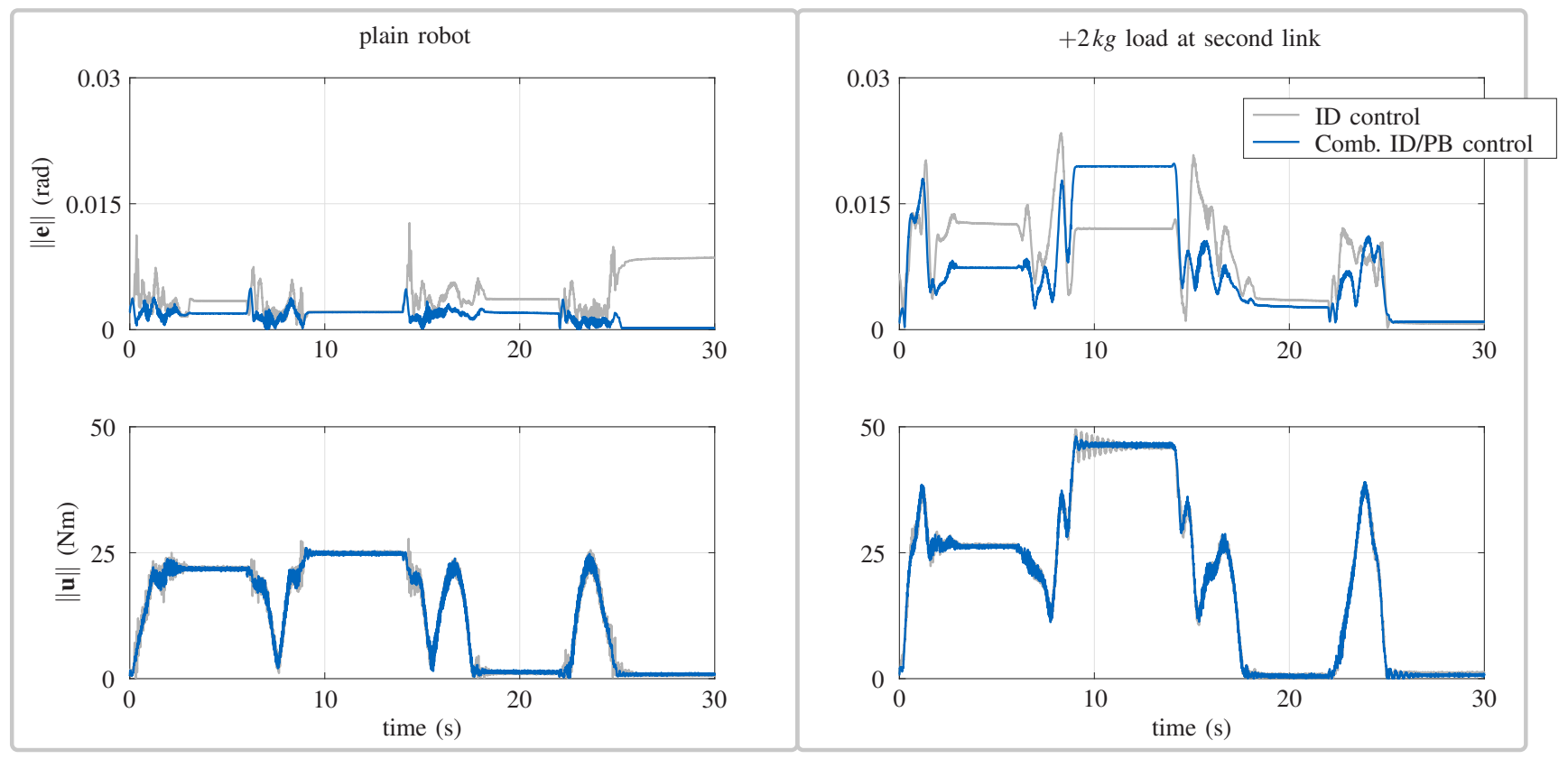

Fig. 7. Experimental results of the trajectory tracking performance of ID control and combined ID/PB control with the assembly of Fig. 5. The test has been executed for two cases: the plain robot (first column of plots) and we emulate a model mismatch by adding $2 \mathrm{~kg}$ weight at the second link (second column of plots).

[6] N. G. Tsagarakis, M. Laffranchi, B. Vanderborght, and D. G. Caldwell, "A compact soft actuator unit for small scale human friendly robots," in Prof. IEEE Int. Conf. on Robotics and Automation, May 2009, pp. 4356-4362.

[7] M. W. Spong, "Control of flexible joint robots: A survey," Coordinated Science Laboratory, University of Illinois, Tech. Rep., Feb. 1990.

[8] A. De Luca and P. Tomei, Theory of Robot Control. Springer-Verlag London, 1996, ch. Elastic joints, pp. 176-217.

[9] M. W. Spong, "Modeling and control of elastic joint robots," Journal of dynamic systems, measurement, and control, vol. 109, no. 4, pp. 310-318, 1987.

[10] M. W. Spong, K. Khorasani, and P. Kokotovic, "An integral manifold approach to the feedback control of flexible joint robots," IEEE Journal on Robotics and Automation, vol. 3, no. 4, pp. 291-300, Aug. 1987.

[11] M. W. Spong, J. Y. Hung, S. A. Bortoff, and F. Ghorbel, "A comparison of feedback linearization and singular perturbation techniques for the control of flexible joint robots," in Proc. American Control Conference, Jun. 1989, pp. 25-30.

[12] A. De Luca, "Feedforward/feedback laws for the control of flexible robots," in Proc. IEEE Int. Conf. on Robotics and Automation, vol. 1, 2000, pp. 233-240.

[13] A. Loría and R. Ortega, "On tracking control of rigid and flexible joints robots," Appl. Math. Comput. Sci, vol. 5, no. 2, pp. 101-113, 1995.

[14] B. Brogliato, R. Ortega, and R. Lozano, "Global tracking controllers for flexible-joint manipulators: a comparative study," Automatica, vol. 31, no. 7, pp. 941-956, 1995.

[15] S. Avila-Becerril, A. Loría, and E. Panteley, "Global position-feedback tracking control of flexible-joint robots," in Proc. American Control Conference (ACC), July 2016, pp. 3008-3013.

[16] M. Keppler, D. Lakatos, C. Ott, and A. Albu-Schäffer, "A passivitybased controller for motion tracking and damping assignment for compliantly actuated robots," in Proc. 55th IEEE Conference on Decision and Control, Dec. 2016, pp. 1521-1528.

[17] A. De Luca, B. Siciliano, and L. Zollo, "PD control with online gravity compensation for robots with elastic joints: Theory and experiments," Automatica, vol. 41, no. 10, pp. 1809-1819, 2005.

[18] A. De Luca and F. Flacco, "A PD-type regulator with exact gravity cancellation for robots with flexible joints," in Proc. IEEE Int. Conf. on Robotics and Automation, May 2011, pp. 317-323.
[19] G. Buondonno and A. De Luca, "A recursive Newton-Euler algorithm for robots with elastic joints and its application to control," in Proc. IEEE/RSJ Int. Conf. on Intelligent Robots and Systems, Sept. 2015, pp. 5526-5532.

[20] — , "Efficient computation of inverse dynamics and feedback linearization for VSA-based robots," IEEE Robotics and Automation Letters, vol. 1, no. 2, pp. 908-915, July 2016.

[21] A. De Luca and L. Ferrajoli, "A modified Newton-Euler method for dynamic computations in robot fault detection and control," in Proc. IEEE Int. Conf. on Robotics and Automation, 2009, pp. 3359-3364.

[22] R. Ortega, A. J. Van Der Schaft, I. Mareels, and B. Maschke, "Putting energy back in control," IEEE Control Systems, vol. 21, no. 2, pp. 18-33, Apr. 2001.

[23] B. Brogliato and C. C. de Wit, Theory of Robot Control. SpringerVerlag London, 1996, ch. Joint space control, pp. 59-114.

[24] A. Giusti and M. Althoff, "Automatic centralized controller design for modular and reconfigurable robot manipulators," in Proc. IEEE/RSJ Int. Conf. on Intelligent Robots and Systems, Sept. 2015, pp. 3268 3275.

[25] R. Ortega and M. W. Spong, "Adaptive motion control of rigid robots: a tutorial," in Proc. 27th IEEE Conference on Decision and Control, vol. 2, Dec. 1988, pp. 1575-1584.

[26] R. Ortega, A. Loría, P. J. Nicklasson, and H. Sira-Ramirez, Passivitybased Control of Euler-Lagrange Systems. Springer-Verlag London, 1998.

[27] A. De Luca, "Decoupling and feedback linearization of robots with mixed rigid/elastic joints," International Journal of Robust and Nonlinear Control, vol. 8, no. 11, pp. 965-977, 1998.

[28] P. Belanger, P. Dobrovolny, A. Helmy, and X. Zhang, "Estimation of angular velocity and acceleration from shaft-encoder measurements," Int. J. Robot. Res., vol. 17, no. 11, pp. 1225-1233, 1998.

[29] P. R. Belanger, "Estimation of angular velocity and acceleration from shaft encoder measurements," in Proc. IEEE Int. Conf. on Robotics and Automation, vol. 1, 1992, pp. 585-592.

[30] M. Wenz, "Automatische konfiguration der bewegungssteuerung von industrierobotern," Ph.D. dissertation, Universität Fridericiana zu Karlsruhe, 2008. 